\title{
CCD PHOTOMETRY: SOME BASIC CONCERNS
}

\author{
C. Sterken $\dagger$ \\ University of Brussels (VUB)
}

\begin{abstract}
We review the status of CCD photometry with emphasis on applications in projects that require considerable amounts of observing time at medium-aperture telescopes and at tasks complementing projects carried out at large telescopes. Associated problems of CCDs are discussed, together with unsuspected difficulties affecting millimagnitude accuracy mainly caused by pixel-size mismatch, cooling, data acquisition, non-linearity of response and improper standardization.
\end{abstract}

\section{INTRODUCTION}

When the photomultiplier (PMT) became in widespread use, its advantages compared to previous detectors, viz. the eye and the photographic plate, led to an explosion of the number of telescopes equipped with photometers for replacing and complementing photographic photometry. Its advantages were clear: high dynamic range, high quantum efficiency (QE), and - if properly applied - a linear response. The principal drawback was its inability to be used for two-dimensional photometry. Now, CCDs have been in widespread use for more than a decade; they essentially offer the same qualitative and quantitative improvements as did the PMT compared to the photographic plate, but CCDs enable us to carry out photometry in two dimensions as well. So, one may expect soon that CCD-based instruments will be the only photometers available at most observatories.

The advantages and problems of CCDs are elucidated in many papers presented at this meeting, and we hear a lot on recent developments in CCD detection. The present paper is not meant to be an exhaustive list of all aspects involved; it is in the first place.intended to remind us of some important points which are relevant to those who use, or intend to use, CCDs for photometry. The paper adresses a broad spectrum of people and overviews the field, but with a restricted approach, namely that of a photometrist trying to determine the magnitude of an object and the color of an object, however, at milli-magnitude accuracy level. This approach is in a very particular framework, namely the framework of an astronomer who does not have large blocks of observing time at a very large telescope, but who belongs to a University Observatory that lacks a staffed software department but has a telescope of the one-meter class or smaller, and is obtaining a one-time grant for acquiring that large, efficient, all-lambda, low dark-current, low-RON four-million pixel chip we hear about at this meeting. That astronomer wishes to complement work done at a large telescope, carry out projects that require considerable amounts of observing time and which cannot be done at a large instrument, do calibrated direct imaging and also time-series photometry at millimag accuracy, and drive the telescope to the edge, to the limit of its possibilities.

$\dagger$ Belgian Fund for Scientific Research (NFWO) 


\section{PRIME ADVANTAGES of CCDs}

Accurate time-resolved photometry is difficult to obtain for objects fainter than about $15^{\text {th }}$ magnitude. It is very true that - even taking into account efficient multi-channel photometers - the high QE of CCDs leads to an important gain in observing time. Photoelectric photometers are almost never scheduled today on telescopes much larger than approximately one meter (Howell 1990). A CCD, moreover, permits simultaneous differential stellar photometry (though it may be difficult to find a good comparison star within the small field of view). Therefore, a major advantage of the CCD is that useful data can be obtained in less-photometric conditions. Also, stars with close companions - for which normal single-channel photometry is difficult - can be measured. Another strong side of the CCD is that it leads to accurate relative magnitudes from point-spread function (PSF) fitting.

For an $11^{\text {th }}$ mag star, a 50-cm telescope like the SAT (Strömgren Automatic Telescope, Florentin Nielsen et al. 1987) barely records 100 counts per second in uvby (half of that amount in Hbeta $\mathrm{N}$ or $\mathrm{W}$, see Fig. 1), and long sequences of integrations are needed to achieve a favorable $\mathrm{S} / \mathrm{N}$ ratio. A CCD camera on the same telescope would do the job in a minute. But the real advantage of the CCD compared to the PMT is in the error budget through the simultaneity of star and sky-background measurement, as seen in Fig. 1, which was constructed with data taken at the moment of strongly varying sky brightness preceding moonset. Note that the data are raw counts per second, not cleaned for any effect of centering $(u, v, b$ and $y$ data were obtained simultaneously, and so were $\mathrm{N}$ and $\mathrm{W}$ in an alternating star - sky sequence with repetitive recentering of the stellar image). The scatter leaves room for doubt, as does the gradient of the descending sky brightness. Careful and well-planned PMT photometry will allow the extraction of high-precision magnitudes and colors for such a moonlit night, but it is clear that a long integration on the star, followed by a short (or even long) integration on the sky will introduce a systematic error. Such problems will not arise in CCD photometry, not even for fainter stars. This advantage of the CCD versus the PMT in fact extends to any application in which a variable sky background might interfere: not only cometary photometry which (by virtue of comets' brightest phases when they approach the Sun) is done during twilight, but also faint-star photometry in external galaxies (LMC/SMC), and even any study of local variations of foreground interstellar extinction.

\section{PRIME DISADVANTAGES OF CCDs}

A first drawback in CCDs is that the integration time of all objects on a single CCD frame is the same, thus the $\mathrm{S} / \mathrm{N}$ for each source is in general not the same all over a single frame. This is a severe limitation for close-binary star programs, where the exposure time must be appropriately short for not overexposing the primary, forcing exposures to be repeated as many times as needed for receiving enough photons from the secondary to obtain an acceptable $\mathrm{S} / \mathrm{N}$ ratio, a situation that is a severe restriction on the potential range of magnitude differences between the components (Van Dessel et al. 1992). A second drawback is the "dead" time caused by reading out the chip and storing the data (about $5 \mathrm{~min}$ for a $2048 \times 2048 \mathrm{chip}$ ). This dead time is the reason why many observers adopt the approach of observing standards during only one very good night (Sinachopoulos 1994, Jønch-Sørensen 1994) and spend little time on extinction measurements. But the most troublesome property of CCDs for accurate photometry is likely to be their high reflectivity - the reflected light getting back to the detector a second time, often in a position where it does not belong (red and blue leaks). That reflection is 

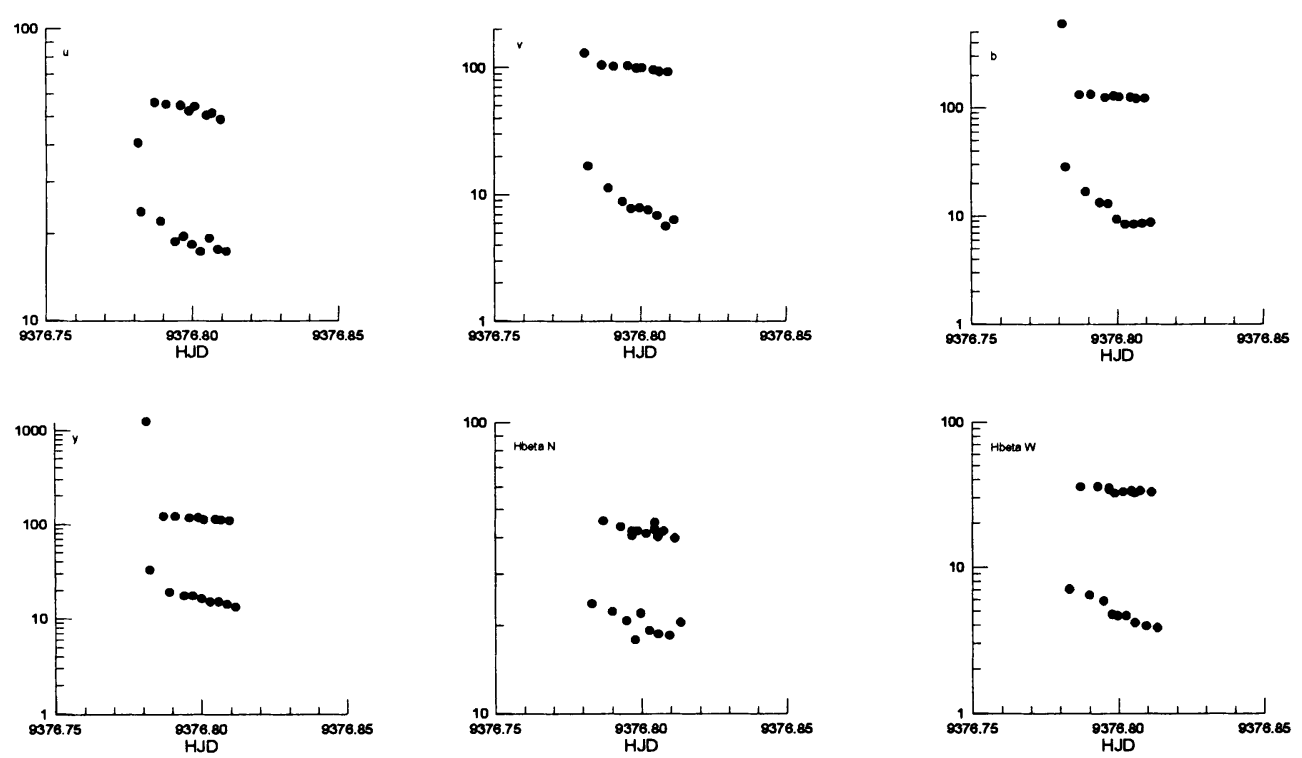

Fig. 1. uvby and $\mathrm{H} \beta$, NW count rates for an $11^{\text {th }}$ magnitude star observed with SAT shortly before moonset. Note the strong decrease in sky brightness during the period of measurement, and also some deviating points

specular, leading to structured ghosts in instruments equipped with CCDs, thus to highly local photometric errors (Tinbergen 1993).

The mayor disadvantage, however, is the huge amount of work involved with data reduction. A general photometric reduction program - that is, to convert PMT and CCD raw instrumental magnitudes to a consistent standard scale - has been distributed by ESO as the PEPSYS context of the MIDAS data-reduction system. It also includes broad introductory information on photometry (Young 1994). But it is the rectification of the raw data frames (shutter-time corrections, removal of cosmic-ray events, bias and dark, flatfielding) and the extraction of instrumental magnitudes that is so time consuming. Walker (1986) states that for a one week observing run one can expect to take at least a same amount of time reducing the data (for extracting a few [ five] stars per frame in uncrowded fields). This agrees with Sinachopoulos (1994), who consecrated about 25 hours of work to obtain the instrumental magnitudes of one night to an internal accuracy of 0.003 . But it is possible to obtain a hundred CCD frames in a night, each frame potentially containing of order 10,000 stars and of order $1,000,000$ individual data-numbers. Stetson (1993) has developed software to carry out these tasks with a minimum of human effort. He can reduce the entire body of data from a typical 
three- or four-night observing run (from raw data frames through the profile-fitting to final, calibrated publishable photometry, see Stetson 1990) in a period of order of three to four forty-hour weeks - still a considerably long time. The real stumbling block thus is the astronomer's time, and we must devote some serious thought very quickly to increasing the efficiency of the data reduction techniques, probably with a view to the automation of as many as the tasks as possible (Sneden 1990). Stetson (1990) advocates the elaboration of comprehensive automatic software for carrying out the numerous reduction steps.

\section{PROBLEMS USING CCDs}

Apart from the particular problems on standardization, there are several problems at millimag accuracy level, viz. how to deal with a varying PSF, and how to deal with the fact that the response inside a pixel is not uniform (non-uniformities of $10 \%$ can arise inside one pixel). It is not unusual to find that about $1 \%$ of the pixels in an array are faulty - they may be of low sensitivity, noisy, dead or have high dark current (Glass 1993).

Besides, observers have to overcome two major obstacles: shutter timing, and twilight flatfielding. There are two shutter timing effects, viz. the (spacially non-uniform) difference between actual and presumed exposure time (a problem that ruins any calibration at millimag level), and the pixel-to-pixel differences (with very adverse effects for short exposures, such as for standard stars). The flatfielding of pixel-to-pixel variations in sensitivity and chip illumination is a fundamental step in reducing CCD frames: its failure will ruin every following step (the pixel-to-pixel variations in sensitivity can amount to a factor of 1.5). Short exposures are unavoidable when observing frames with standard stars. Hainaut et al. (1994) avoid this by exposing defocused standard-star images for $10-20 \mathrm{sec}$, and estimate overexposure due to shutter delay to be $0.5 \pm 0.3 \mathrm{sec}$ (the real value could, however, not be determined).

Twilight flatfielding poses two problems:

a) Short exposures are inevitable - and the two-dimensional flatfield structure will be significantly altered by the shutter function at exposure times of a few seconds and less. ${ }^{1}$ Thus, with a shutter delay of the order of $0.5 \mathrm{~s}$, also a flatfield exposure shorter than $5 \mathrm{sec}$ will be systematically affected on the level of more than $10 \%$.

b) Only very little time is available for taking the frames - the readout overhead is not less than for science exposures, and this leads to only a fraction of effectively useful twilight time (too dark skies bring stellar images on the CCD, too bright skies saturate the detector). Though the short-exposure problem decreases when using narrow-band filters, Jønch-Sørensen (1994) points out that getting enough sky flatfields with appropriate intensity levels in six filters requires many nights. Note that the importance of correcting for the shutter effects in twilight flats will increase with the larger CCDs because of their long read-out times.

Fig. 2 illustrates the rate of change of sky surface-brightness level, as measured on two nights using the SAT (uvby simultaneously, $\mathrm{H} \beta$, NW simultaneously). As the insert shows, there is a slight wavelength dependence in the slope of the change (the deviating N,W data are

${ }^{1}$ but also by the difficulty of discriminating cosmic-ray events. 
from a different night, both yield exactly the same slope). The discontinuity in uvby is due to the removal of a neutral-density filter, and it illustrates that the time available for taking twilight flatfields can be considerably extended using stacked or wedged neutral-density filters. There is a substantial curvature during the "bright" part of twilight, an effect that is partly due to the dead time of the system (such curvature, however, can also have a physical cause, such as the presence of volcanic dust in the atmosphere). However, the non-linearity seen in these curves is not a problem of PMT-based photometry alone! Saturation effects in CCDs may produce similar symptoms: Hodapp et al. (1992) report an excess dark current which follows the detected pattern of a previous strong exposure. Although its effects are negligible after a few complete reads of the device for normal background-limited exposures, it can remain a nuisance for up to an hour for exposures with low backgrounds. There is also a recently-discovered nasty problem (Fosbury 1994), viz. the fact that charge bleeding in columns seems to alter the bias level, and this change depends on the intensity of the brightest star that causes the charge bleeding. That problem, of course, not only affects the flatfield frames, but also the science exposures.

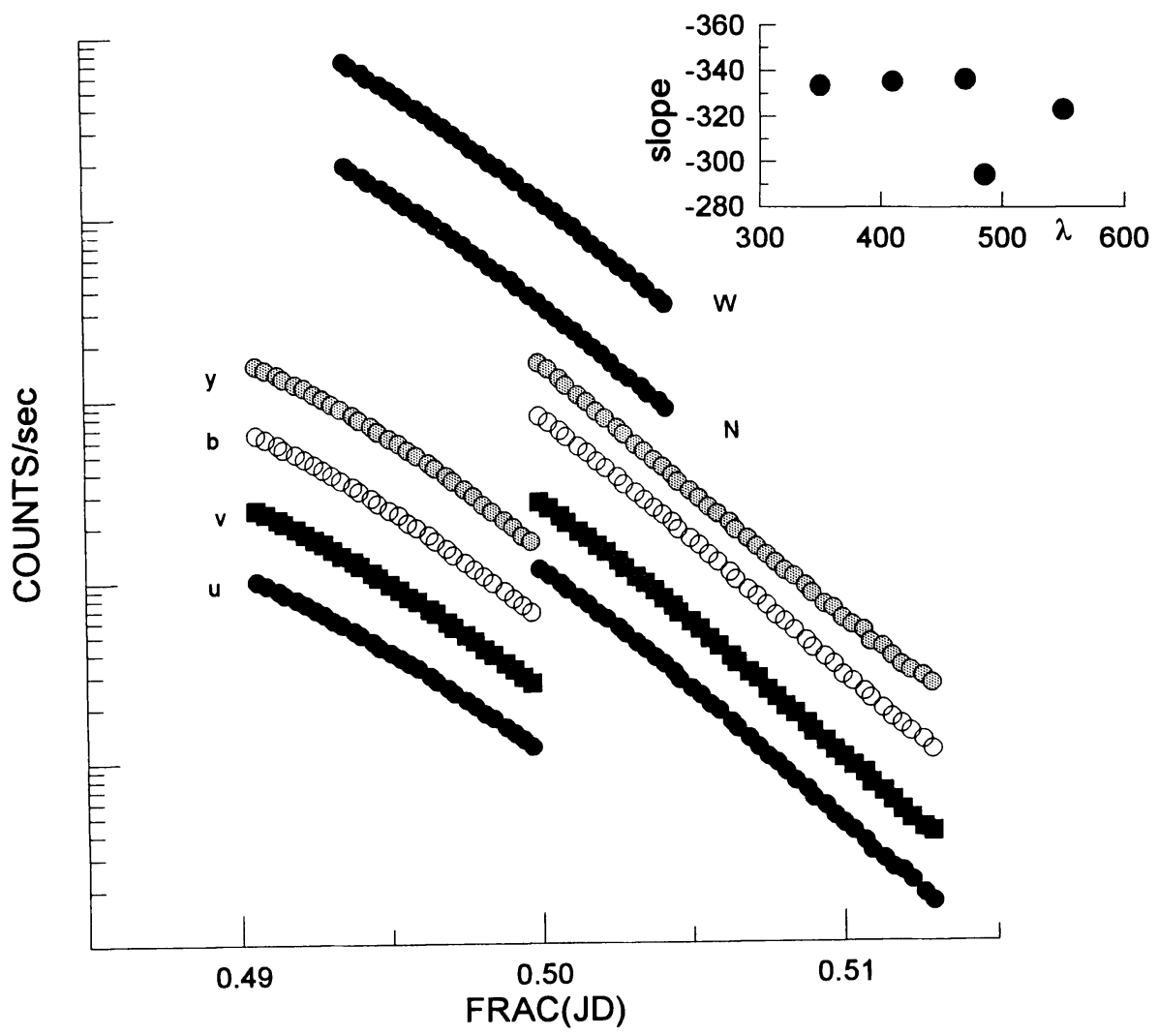

Fig. 2. Log of the twilight sky brightness for uvby and NW (the latter were obtained the night after the uvby measurements. All count rates were shifted along the vertical axis. The upward jump in uvby is due to the removal of a neutral-density filter. The insert shows the wavelength dependence of the slope of the sky brightness variation (log counts per day) 
Tyson and Gal (1993) have derived a very useful expression for calculating an optimal time series of flatfield exposures adjustable to any given CCD read-out time. They give tables that allow all frames to record similar count levels - their data are taken at the zenith, which is the case for most flatfields. Surma (1993) develops a simple method to deconvolve the intrinsic flatfield and the two-dimensional shutter function from a series of flats of different exposure times. Using these intrinsic calibration functions it is possible to flatfield long-exposed object frames accurately and to construct flatfields which include a specified shutter contribution and thus are valid for any given short exposure time in an object exposure.

Tobin (1993) discusses CCD flatfielding using an illuminated dome screen: despite uniform screen illumination, non-flat response results from telescope flexure and by light scattered off by telescope baffles: a $0.3 \%$ accuracy is achievable. An extremely important matter is that beams from lamps should resemble beams from the telescope, and they should therefore have the same focal ratio, and proceed from a pupil at the same distance and with the same central obstruction ('Tinbergen 1993).

\section{PITFALLS EMPLOYING CCDs}

CCD cameras, sometimes cheap commercial models, are often installed at small (Schmidt-Cassegrain) telescopes at university (and private) observatories on account of the availibility of these telescopes, and because of the fact that on these telescopes a very large amount of observing time is obtainable. Such simple systems, often with standard imaging and reduction software, can lead to deterioration of precision in some cases. But even high-tech professional implementations are prone to systematic disturbances: one is, for example, parasite light caused by LEDs. Other disorders are as well due to misconceived construction details as to mistaken views of application.

\subsection{Pixel Size}

The small size of some chips tempts the use of a scale which maximises the sky coverage (for variable stars, where one seeks to observe a comparison star on the same frame) but which leads to undersampling. This is particularly serious when there is dead space between pixels or uneven sensitivity within pixels. To cover star images with several pixels and to fully utilise any good seeing, a pixel size no greater than 0.5 is required. With pixel sizes ranging from 20 to $30 \mu$ that implies a focal length of $10-15$ meters. Photometric accuracy falls off rapidly as the star images become undersampled; for work that does not require the small pixel size (e.g. galaxy surface photometry) shorter focal lengths can be used (Walker 1986). Another point is that CCDs are now approaching the size of small photographic plates, and since the FWHM can be $10 \%$ larger at the corner of a 13 arcmin square field (CTIO 0.9-m f/13.5 telescope, Walker 1993), a field flattener is needed to avoid a strongly varying PSF as a function of field position. The same problem can occur if the CCD chip itself is warped.

\subsection{Cooling}

At large observatories, detector cooling is achieved by liquid gas cryogenics or by Peltier cooling. At smaller installations, only the latter - or no cooling at all - is the rule (note that these Peltier elements can be small in size, but are inadequate for installing inside the tube of a Schmidt telescope). As is the case with PMTs, the dark current is a function of the detector's 
working temperature. So, Jønch-Sørensen (1994) found nightly variation of the bias level when the temperature control was occasionally defective.

It is well known that the temperature in a dome fluctuates rather irregularly during the night, and - though the fluctuations are an order of magnitude smaller - the temperature inside an apparatus often varies in a way uncorrelated with dome temperature. This is illustrated in Fig. 3, which gives the temperature measured in the SAT dome and inside the SAT photometer during four nights: (a) shows the trend of the temperature variation at the detector and at two different measuring points in the dome, (b), (c) and (d) compare dome temperature and detector temperature at the same time resolution as the scientific measurements. Whereas (a) and (b) exhibit a fairly linear behavior for the detector temperature, this is not so in the other cases, where a non-linear trend with superposed strong fluctuations (with a gradient of $0^{\circ} 2 / \mathrm{min}$ ) is seen. Our data show that the simple approach of measuring dome temperature at the beginning and at the end of a night and applying some kind of correction linearly with time is a totally unfounded procedure. Let us note that dome temperature usually undergoes a steep drop during the first hour of the night, and that the pattern of variability of dome temperature is strongly dependent on the type of observations carried out: time-series photometry, with dome orientation that only slowly changes in time yields a different temperature pattern than does all-sky photometry with its higher frequency of dome rotation. A cooling system must be able to correct for such fast changes. Tyson (1990) points out that stability is crucial if imaging at 100 parts in $10^{6}$ of the night-sky background is required (not only for what concerns temperature in particular, but for CCD stability in general).
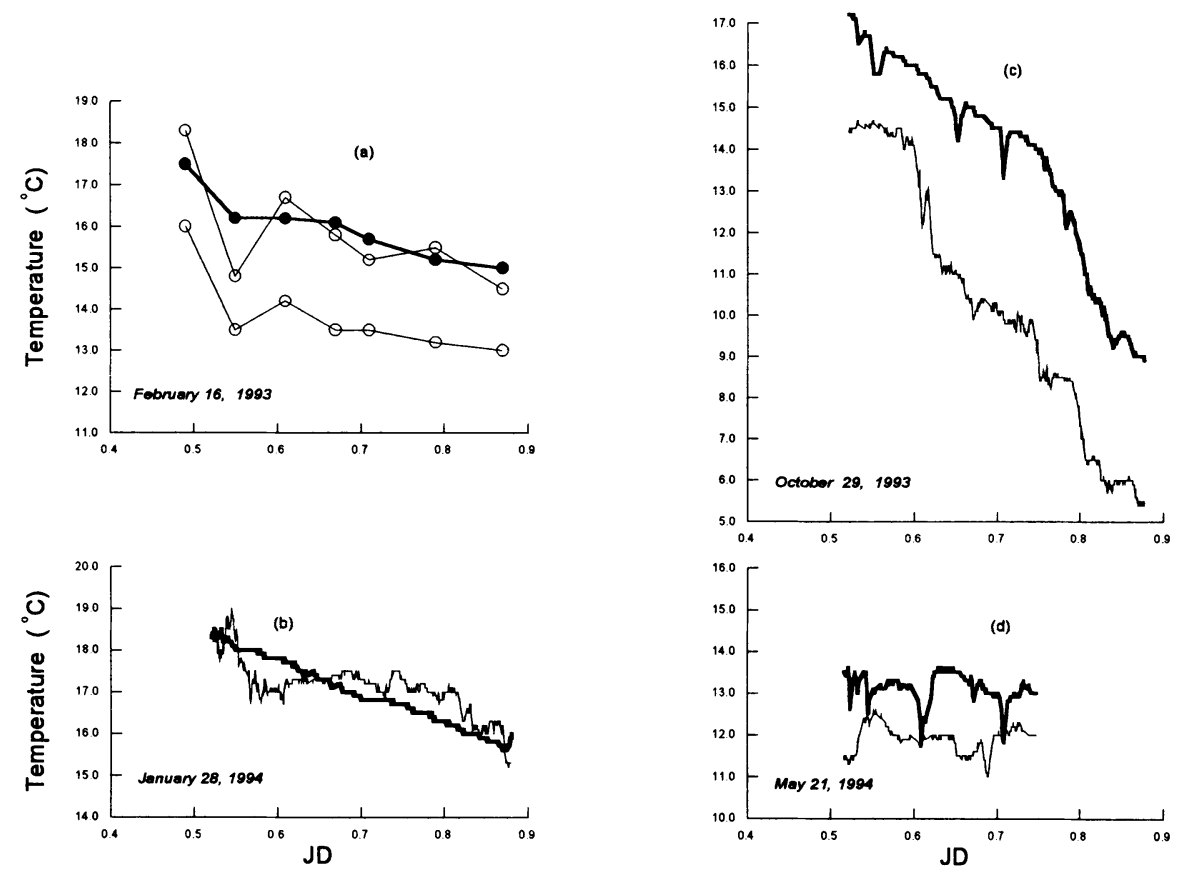

Fig. 3. (a) depicts the run of dome temperature (open circles) measured at two different locations in the dome, and detector temperature (full circles). (b), (c) and (d) give dome temperature (thin line) and detector temperature (thick line) for three other nights 


\subsection{Data Acquisition at the Telescope}

Many papers deal with data reduction software, but what about bug-like errors that are generated at the time of observation, and that may accumulate to large effects? One such problem already occurs when the telescope control system (TCS) is directly linked to the data acquisition system (DAS). In such cases, the telescope coordinates are recorded in the headers of the data files, and the person who carries out the data reduction (often a student who is not familiar with the instrumental configuration) is tempted to use these recorded coordinates as absolute coordinates. Such procedure imports errors due to the more or less frequent application of the "common correction" (CC) routine - that is, an instruction that corrects for the difference between the actual telescope pointing and corrected catalogue coordinates -leading to repetitive introduction of a non-rigid coordinate system for the objects measured, especially when a CC is done during every cycle of measurement of program and comparison stars when monitoring with an automatic or semi-automatic telescope. The effect is even more dramatic for TCS systems that apply approximate formulae for precession corrections and, as such, introduce large errors for objects with large positive or negative declination (the error will also appear when small mechanical shifts or oscillations in $\alpha$ or in $\delta$ occur). The consequence is illustrated in Fig. 4, where the differences $\mathrm{X}-\mathrm{X}_{\text {tel }}$ of the airmass calculated with catalogue coordinates and telescope coordinates (data obtained with the SAT) is plotted in function of airmass. The top figure gives the result for two low-declination stars a few arcminutes apart (as could appear on a same CCD frame) that have been measured sequentially for several hours. For airmasses below 1.7, the difference $\mathrm{X}-\mathrm{X}_{\text {tel }}$ amounts to not more than $0^{\mathrm{m}} 001 \mathrm{mag}$, and does not influence the accuracy at millimag level. The other points all refer to two stars at declination $\sim-70^{\circ}$ and separated by several degrees. They were observed sequentially, and every four to five minutes a CC command was given. Besides the two curved sequences, there are a number of dispersed points which correspond to moments when the autocentering failed for some reason, or when a reboot of the TCS occurred. In differential photometry, as illustrated in Fig. 4, pseudo airmass-differences amounting to 0.15 may be generated, a situation that may lead to breakdown of any photometry even at the centi-mag accuracy level.

\subsection{Non-linearity of Response}

Most CCDs appear to be linear to at least $0.1-0.5 \%$ from very low signals to at least $80 \%$ of the full well capacity (Walker 1993). A surprising number of CCD instruments turn out to have non-linearity and other problems of the sort long familiar to photographic photometrists (Young 1994). Schwartz and Abbott (1993) describe non-linearity of response amounting to $4 \%$ due to parasite noise in the analog-to-digital converter board. However, linearity is generally assumed on the basis of absence of adverse effects, but no vigorous linearity tests seem to be carried out, and it would be highly valuable if all observing sites provided a robust linearity-check routine at the telescope (Jønch-Sørensen 1994).

\section{EXTINCTION AND STANDARDIZATION}

Extinction determination is, in principle, in no way different for CCD data than for PMT measurements. Building transformations to a standard system is a similar job. Still, virtually no CCD photometrists derive extinction directly from their own data. They spend a minimum of time on technical observations (remember, frequent observations of standards all over the sky 


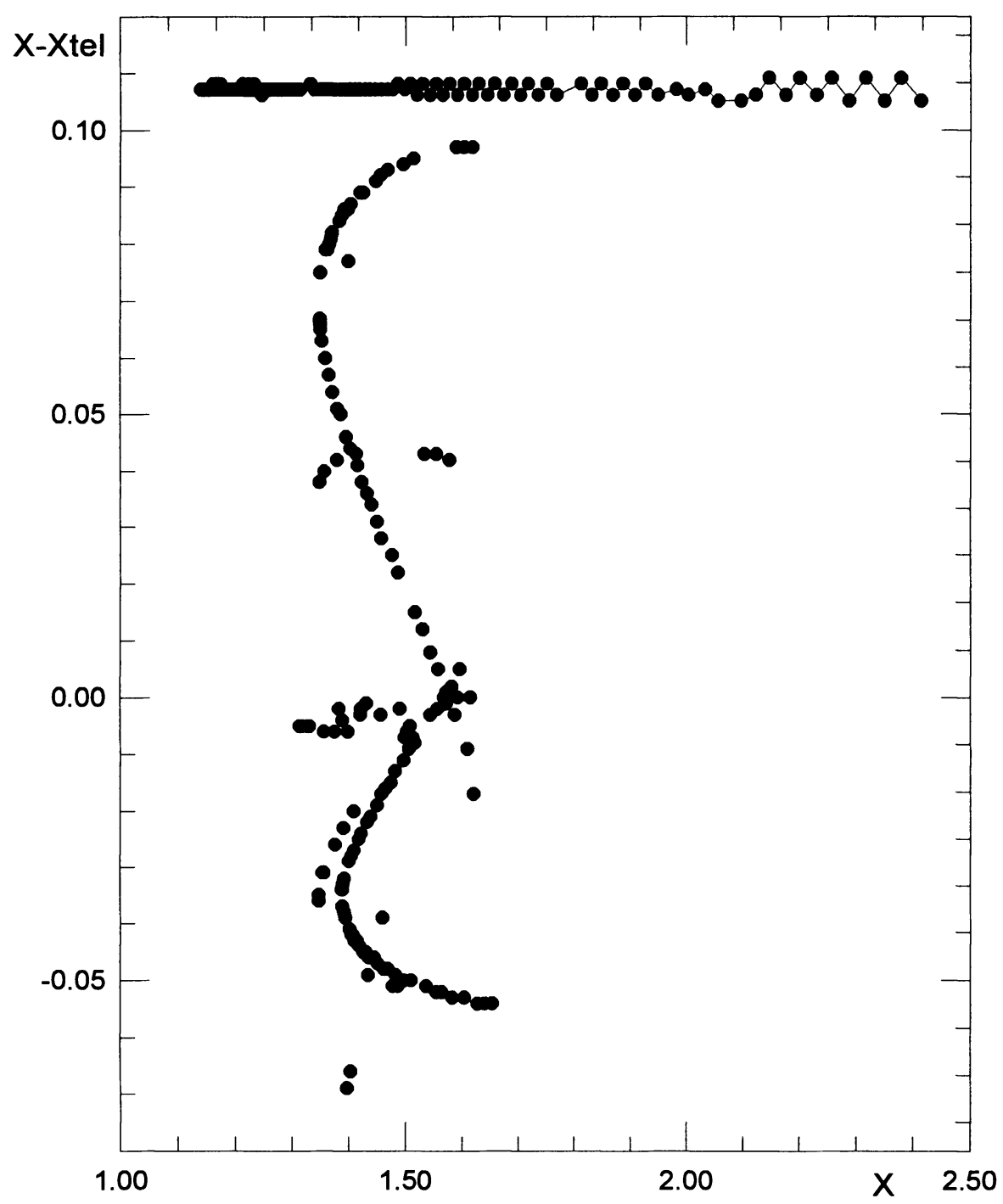

Fig. 4. Difference in airmass using (accurately precessed) catalogue coordinates, and coordinates recorded by the telescope control system. The top curve is for two very close low-declination stars, bottom curves are for two stars several degrees apart at $\delta \sim-70^{\circ}$ (see text) 
also demand a fast-slewing and fast-setting telescope), and supplementary information on extinction from a nearby small $(50-\mathrm{cm})$ telescope is often sought (Hainaut et al. 1994, Jønch-Sørensen 1994). Taming atmospheric extinction will require frequent short observations of standards all over the sky and a means of monitoring extinction variations near the object being observed (Tinbergen 1993). The response curves of CCDs vary much more than those of PMTs (also, CCDs have an extended red response, and their blue response falls steeply). Thus, CCD photometric passbands poorly match existing standard systems ${ }^{2}$ and, in principle, each CCD chip would need its own custom-designed filters, a solution that cannot be straightforwardly considered for every CCD camera.

Fig. 5 illustrates acquired accuracies from two sources of photometry. For faint stars the (internal) errors are not dominated by photon statistics but by the accuracy of the flatfielding, the quality of removing cosmic ray events and $C C D$ defects and the quality of the profile fitting. When it comes to calibration, and transformation to a standard system, several additional systematic errors may be introduced. First of all, exposure times may strongly differ with passband (see Fig. 4), so that for a same star a blue frame is shutter-time correct, while a red frame may be, at the same time, affected with errors of several per cent. Such situation ruins the accuracy of the associated color index; the color index, in turn, introduces the damage directly in the transformation equations. Furthermore, standards as faint as the objects under study are not available (standards with a good range in color should be on a single CCD frame), so one must either use short exposures, or recur to defocused images. Defocusing not only ruins surface - or crowded-field photometry, it also changes the light path, as is stressed by Walker (1986): “ ... the defocusing of bright stars is not in general to be recommended since one of the major advantages of the CCD is that the standard star frames and the program star frames can be measured in precisely the same way. Any departure from this must introduce a systematic error at some level". Young (1994) underlines that there must be angular effects in CCDs, which should show up in defocused images: the response depends on angle as well on position. A pixel in an out-of-focus image receives light from only a small part of the pupil. But in flatfields, it sees the whole pupil. These effects, at least, should vary according to the Fresnel

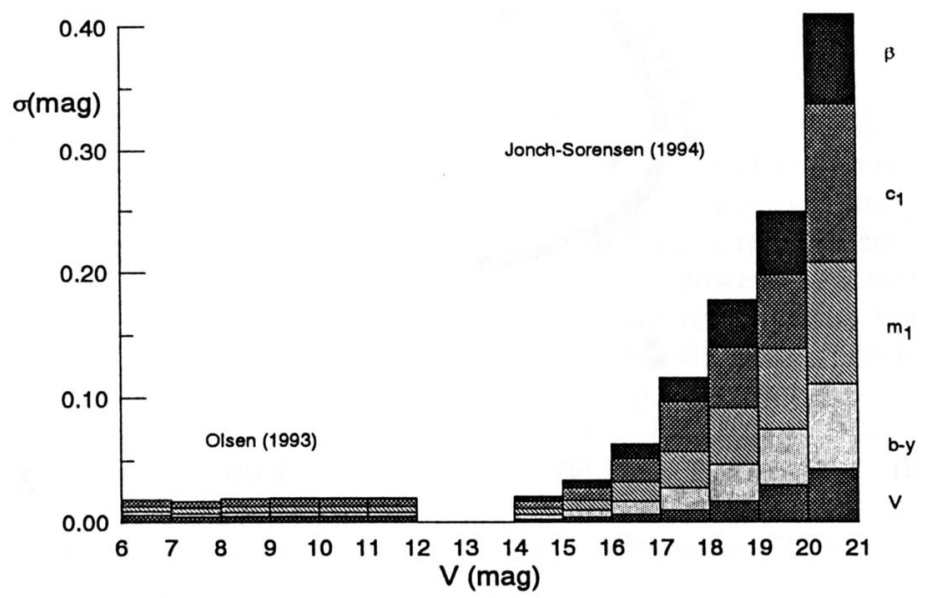

Fig. 5. Standard deviations for uvby (Olsen 1993, PMT, SAT telescope) and uvby $\beta$ (Jønch-Sørensen 1994, CCD, Danish 1.5-m telescope)

see Young's paper in this conference 
reflection coefficients in back-illuminated CCDs, and should be larger due to shadowing by electrodes in front-side illumination.

Let us stress, however, that most of the standard star work at SAAO has been carried out with the $0.5-\mathrm{m}$ telescope, and much of the success of the programs was due to the large amount of observing time available at the telescope (Menzies 1993). It is clear that a repetition of such enterprise at a larger telescope is impossible today. Menzies suggests that new CCD-sized fields in the $\mathrm{E}$ regions would be the best option as far as accurate transfer of zero point and color scales is concerned, but it will be a very time-consuming exercise to find suitable fields containing a reasonable number of stars per CCD frame.

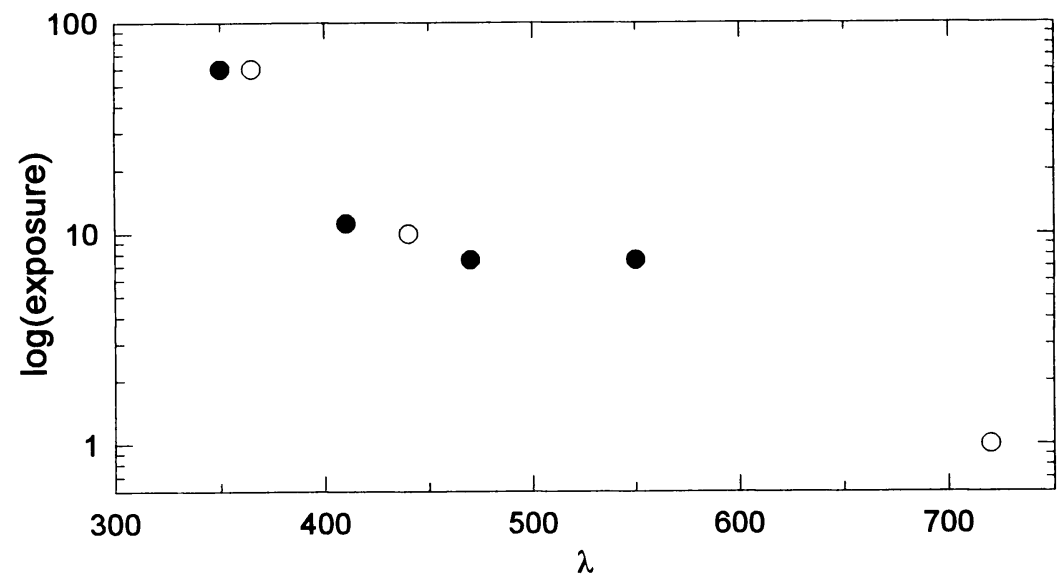

Fig. 6. Normalised exposure times (in seconds) for CCD frames: filled circles from Jønch-Sørensen (1994), open circles from Surma (1993)

Part of the problem of standardization is, of course, caused by the sometimes very short lengths of the observing blocks at large telescopes. As Hainaut et al. (1994) put it: "it is essential to have complete nights instead of half-nights, because sharing the nights makes it very difficult to obtain a sufficient number of calibration frames".

An aspect directly related to standardization is a realistic determination of the flux threshold. The high QE of CCDs has enticed users to press to the faintest possible limits, where systematic errors can lie hidden in the sky noise (Young 1994). The faintest flux level achievable in a given integration time depends on the number of exposures, the CCD and background noise and systematics, and the details of the detection and filters. At the faint limit, there is a tradeoff between angular resolution, confidence of detection, and limiting surface brightness. The limiting magnitude in any given band is also different for the various degrees of filtering of the data: detection, evaluation, splitting, classification, or photometry (Tyson 1988). A very interesting pilot program has been undertaken by Hainaut et al. (1994), who tried to push the performance of the ESO NTT to its limit by searching for comets beyond heliocentric distance $r \sim 10 \mathrm{AU}$ (in uncrowded areas, $|\mathrm{b}|>15^{\circ}$ ). During three half-nights they used a 1024 x 1024 TK1024M chip, and applied a very tedious procedure of data reduction. 
However, no candidate cometary images were found on any of the frames. It should be stressed that in such programs, "non-detection" must be considered as a real data point, and that the derived "limiting magnitude", with its intrinsic mean error, is directly used for putting constraints on proposed models.

\section{CONCLUSIONS}

We have highlighted some procedures and pitfalls of CCD photometry. Many a CCD observer does not achieve a complete photometric standardization of the data, but relies on the support of nearby small telescopes, in a way very similar to relying on smaller telescopes to get the best possible ephemerides for moving objects. These facts show that observers are convinced of the fact that their time on large telescopes is too expensive to spend it on accurate standardization and extinction, an attitude that only gives further support to the idea that it is cost effective to have an additional $0.5-\mathrm{m}$ telescope dedicated to monitoring the extinctione over the sky during the night (see also Penny 1993).

In fact, the above also shows that, when working to millimag accuracy levels in photometry, too much is left to the individual observer, such as scheduling of extinction and standard star measurements, and calibration of the instrument. To achieve good photometry, not only suitable standard sequences must be available, but also standardized operation in the dome and at the workstation afterwards is essential to get data of good quality and homogeneity (Tinbergen 1993).

In this context is the increasing occurrence of closing small telescopes for reasons of economy when building large ones - causing large scientific loss for little gain - resembling more than anything else a situation of being invited to a great dinner where the host is saving on salt and pepper.

\section{REFERENCES}

Florentin Nielsen, R., Norregaard, P. and Olsen, E.H. 1987 The Messenger 50, 45

Fosbury, A. 1994, private communication

Glass, I. S. 1993 in Stellar Photometry - Current Techniques and Future Developments,. C. J. Butler and I. Elliott, eds., Cambridge University Press, Cambridge, p.154

Hainaut, O., West, R. M., Smette, A. and Marsden, B. G. 1994 A\&A 289, 311

Hodapp, K. -W., Rayner, J. and Irwin, E. 1992 PASP 104, 441

Howell, S. B , 1990 in CCDS in Astronomy. II., A. G. Davis Philip, D. S. Hayes and S. J. Adelman, eds., L. Davis Press, Schenectady, p. 133

Jønch-Sørensen, H. 1994 A\&A 292, 363

Menzies, J. W. 1993 in Precision Photometry, D. Kilkenny, E. Lastovica and J. W. Menzies, eds., SAAO, p. 87

Olsen, E. H. 1993 A\&AS 102, 89

Penny, A .J., 1993, in Stellar Photometry - Current Techniques and Future Developments, C. J. Butler and I. Elliott, eds., Cambridge University Press, Cambridge, p. 146

Schwartz, H. E. and Abbott, T. M. C. 1993 The Messenger 71, 53

Sinachopoulos, D. 1994, personal communication

Sneden, C. 1990 in CCDS in Astronomy. II., A. G. Davis Philip, D. S. Hayes and S .J. Adelman, eds., L. Davis Press, Schenectady, p. 221 
Stetson, P. B. 1990 in CCDS in Astronomy. II., A. G. Davis Philip, D. S. Hayes and S. J. Adelman, eds., L. Davis Press, Schenectady, p. 71

Stetson, P. B. 1993 in Stellar Photometry - Current Techniques and Future Developments, C. J. Butler and I. Elliott, eds., Cambridge University Press, Cambridge, p. 291

Surma, P. 1993 A\&A 278, 654

Tinbergen, J. 1993 in Stellar Photometry - Current Techniques and Future Developments, C. J. Butler and I. Elliott, Cambridge University Press, Cambridge, p.130

Tobin, W. 1993 in Stellar Photometry - Current Techniques and Future Developments, C. J. Butler and I. Elliott, eds., Cambridge University Press, Cambridge, p. 304

Tyson, J. A. 1988 AJ 96, 1

Tyson, J. A. 1990 JOSA 7, 1231

Tyson, N. D. and Gal, R. R. 1993 AJ 105, 1206

Van Dessel, E., Sinachopoulos, D. and Prado, P. 1992 in IAU Colloquium No. 135, Complementary approaches to Double and Multiple Star Research, ASP Conf. Series 32, 362

Walker, A. R. 1986 in IAU Symposium No. 118, Instrumentation and Research Programmes for Small Telescopes, J. B. Hearnshaw and P. L. Cottrell, eds., D. Reidel, Dordrecht, p. 33

Walker, A. R. 1993 in IAU Colloquium No. 136, Stellar Photometry - Current Techniques and Future Developments, C. J. Butler and I. Elliott, eds., Cambridge University Press, Cambridge, p. 278

Young, A. T. 1994 Reports on Astronomy, XXIIA, 229

\section{DISCUSSION}

CULLUM: At what level do errors due to dome flatfields (for example due to CCD fringing and the different optical paths for measurements and calibrations) occur?

STERKEN: The best paper on this topic is Tobin's Dublin paper. I believe he reaches $0.3 \%$ accuracy.

YOUNG: Temperature effects in filters are more important than in detectors, but are rarely either measured or controlled.

STERKEN: Yes, especially in glass filters, as you showed decades ago!

YOUNG: Using a different telescope to measure extinction introduces the problem of transforming between the two instrumental systems.

STERKEN: That is right, the extinction correction is a transformation in itself.

PENNY: A flatfield on the sky has illumination flat to about $1 \%$, due to scattered light in the telescope. This can be seen by comparing dusk and dawn sky flats, where the bright part of the sky is on opposite sides of the telescope.

YOUNG: Twilight flats can be dangerous, because the spectral distribution of twilight is very different from that of stars, particularly because of ozone absorption.

STERKEN: So is the case of a dome flat: the incandescent lamp will rarely match the energy distribution of a star, or that of a galaxy. 
VERSTRAELEN: You mentioned the importance of pixel size to avoid under- or oversampling. Can you give an upper and lower limit for the pixel size to obtain a well sampled image?

STERKEN: It depends on the seeing, but also on the quality of optics: if the image field is not flat (as in the case of a fast Schmidt-Casseggrain telescope), image sizes at the edges will be larger than in the center, thus undersampling may happen in some areas, and in others not. Half a second of arc is often a guideline for an upper limit.

BESSELL: KPNO recommends using stacked median filtered sky from all exposures during the night for flatfields. Can you comment on this?

WALKER: This technique in general works well. I have found it useful to smooth the resulting frame and use it as a correction to a twilight flat. If you want to use the frame directly you must have plenty of sky, otherwise the $\mathrm{S} / \mathrm{N}$ in the flatfield will be poor.

FLORENTIN-NIELSEN: Many telescopes have not got proper baffling of the optics. To what extent does that affect the phometric accuracy?

STERKEN: Improper baffling, especially for open-tube type telescopes may lead to deterioration of accuracy due to stray light from the dome (computer displays and some lighting) or from the Moon. One can calibrate the effect by performing sky measurements while modulating the light source in the dome, though one should not forget that there is also contamination by light leaks in the photometer housing.

TOBIN: I'd just like to reiterate that producing a flat flatfield is not trivial. Initially our dome and sky flats agreed to better than $1 \%$, but moving a star around the chip showed the flats were both warped by several percent. Annular baffling to reduce scattered light is very important. 